\title{
Knowledge sharing and language diversity in organisations: influence of code switching and convergence
}

\author{
Farhan Ahmad* and Gunilla Widén \\ Information Studies, \\ Faculty of Social Sciences, Business \& Economics, \\ Åbo Akademi University, \\ Fänriksgatan 3B, 20500, Turku, Finland \\ Email: farhan.ahmad@abo.fi \\ Email: gunilla.widen@abo.fi \\ *Corresponding author
}

\begin{abstract}
Individual-level knowledge sharing is an important collaborative activity that is critical for organisational performance. As multilingual workplaces are becoming common, it has become increasingly important to understand the impact of language on knowledge sharing. Although previous research on knowledge management acknowledges the influence of language on knowledge sharing, the language use (practices) that actually conditions this effect remains largely unexamined. In this paper, we introduce two types of language practices known as code switching and convergence in sociolinguistics. By using insights on language from sociolinguistics, we attempt to show how code switching and convergence by organisational employees may influence individual-level knowledge sharing in multilingual organisations. We also suggest some new research directions for language and knowledge sharing in both theoretical and methodological terms. Understanding the influence of code switching and convergence on knowledge sharing is one step toward a better understanding of knowledge sharing as a whole in multilingual organisations. It would enhance the odds of developing knowledge management strategies that may neutralise or at least limit the negative influence of language diversity on knowledge sharing.
\end{abstract}

Keywords: knowledge sharing; language diversity; sociolinguistics; multilingual organisations; multinational organisations; knowledge management; corporate language; code switching.

Reference to this paper should be made as follows: Ahmad, F. and Widén, G. (XXXX) 'Knowledge sharing and language diversity in organisations: influence of code switching and convergence', European J. International Management, Vol. X, No. Y, pp.xxx-xxx.

Biographical notes: Farhan Ahmad is research associate in information studies at Åbo Akademi University, Finland. He received his master's degree in international management from Stockholm University, Sweden. In his doctoral dissertation, he examines the influence of language on knowledge sharing behaviour in multilingual workplaces. His research interests are knowledge sharing, language diversity, multilingual organisations and sociolinguistics.

Gunilla Widén is Professor of Information Studies at Åbo Akademi University. She received her PhD in Information Science in 2001. During 2004-2005 she was visiting researcher at School of Computing, Edinburgh Napier University. 


\section{F. Ahmad and G. Widén}

She is Docent in Information Management at University of Tampere. Her research interests are within the areas of information behaviour, knowledge management, social capital and knowledge sharing. She has lead two large research projects financed by Academy of Finland investigating key skills in information society as well as various aspects of social media and a changing information behaviour (2006-2014). She has published widely in her areas of expertise and been appointed expert in several evaluation committees.

\section{Introduction}

In today's competitive business environment, knowledge is known to be the most critical resource for the success of an organisation. An organisation's ability to outperform its competitors depends to a great extent on how efficiently it manages its knowledge resources. Inherently, knowledge resides within the individuals who are responsible for the creation, application and transformation of knowledge into work routines and processes (Bock et al., 2005; Nonaka and Konno, 1998). How individuals collaborate and share their knowledge with each other has direct consequences for organisational learning and innovation (Radaelli et al., 2011). There is no doubt that knowledge sharing is a very important knowledge-centered activity, although it is also a complicated behaviour sensitive to a number of individual, organisational and contextual factors (Gressgård, 2015; Yesil, 2014; Wang and Noe, 2010). Recently, language diversity has been acknowledged as an important and influential factor in knowledge sharing in multilingual organisations (Ahmad and Widén, 2015). The workforce of the organisations has become increasingly multilingual in the last decade, owing to the globalisation of business and international immigration. Linguistic differences are well known for their potential to disrupt social interaction (Mcall, 2003). Therefore, this recent change in the organisational workforce has aroused the interest of researchers and practitioners in language and its influence on knowledge sharing in multilingual contexts (e.g., Ahmad and Widén, 2015; Klitmøller and Lauring, 2013; Makela et al., 2007; Tan and Gartland, 2014; Welch and Welch, 2008).

A review of the previous knowledge-sharing literature shows that the influence of language on knowledge sharing in organisations has been studied mainly in terms of language proficiency, language commonality, and language symbolism. Under the perspective of language proficiency, research proposes that lack of linguistic skills in a certain language, particularly the corporate language, may lead to isolation from knowledge-sharing networks, inability to share worthy ideas with others, and misunderstandings in knowledge communication (Klitmøller and Lauring, 2013; Makela et al., 2007; Welch and Welch, 2008). Language commonality is another perspective on language where the extent of similarity or difference between languages in multilingual workplaces is the point of analysis in relation to knowledge sharing. Language commonality influences knowledge sharing because it develops shared vision and mutual understanding between knowledge-sharing participants (Evans et al., 2012; Henderson, 2005; Schomaker and Zaheer, 2014). In language symbolism, the relationship between language and knowledge sharing is analysed in the context of language's association with other social forces such as power, identity, and culture. For example, perceiving identity 


\section{Knowledge sharing and language diversity in organisations}

and cultural similarity through language in multilingual workplaces brings certain language speakers closer, leading to limited knowledge sharing across linguistic boundaries (Ahmad and Widén, 2015; Tange and Lauring, 2009; Lauring and Tange, 2010). The separation between these three views on language in knowledge-sharing literature is neither absolute nor unambiguous. Indeed, these three views are related to one and another, but this division does provide a useful holistic view of the current state of language and knowledge-sharing research.

In an exhaustive review of the knowledge-sharing literature, Foss and colleagues (2010, p.461) suggest that a general trend in studies analysing the relationship between different factors and knowledge sharing has been to focus on "macro-macro links" whereas little attention is paid to "micro level processes that mediate between macro variables." For the broader knowledge-sharing literature, the validity of this claim is subject to discussion. However, in the context of knowledge-sharing research dealing with language in multilingual organisations, the argument seems to hold water. Language as a concept can be approached in a number of ways both at the micro and the macro level (Moyer, 2008). In many of the previous studies on language and knowledge sharing in multilingual environments, language has been approached at the general macro level. It has been taken as a social factor, and its influence on knowledge sharing between employees has been studied through general macro constructs, as mentioned above. However, we do not see any comprehensive knowledge-sharing study where the influence of the employees' language use on knowledge sharing in multilingual organisations has been examined. Language use refers to the ways people deploy their linguistic resources in social interactions. The focus on language use has the potential to bring to the fore linguistic behaviours that have consequences for knowledge sharing. In order to develop a comprehensive and better understanding of the influence of language on knowledge sharing, language should not only be approached at the macro but also the micro level, that is, language in use.

In this paper, we introduce two types of language practices called code switching and convergence from sociolinguistics and show how they influence and relate to individuallevel knowledge sharing in multilingual organisations. The purpose of this paper is twofold: (a) to illustrate the influence of language-use practices (switching and convergence) on knowledge sharing; and (b) to outline directions for future research on knowledge sharing and language in multilingual organisations.

The paper is structured as follows. First, we discuss the importance of language use, its potential to offer useful insights into the relationship between language and knowledge sharing and reasons for choosing code switching and convergence from sociolinguistics. After this, we introduce code switching and convergence and analyse their respective relationships with knowledge sharing between employees in multilingual organisations. Finally, we outline some theoretical and methodological suggestions for future research on language and knowledge sharing and then conclude the paper.

\section{Language use}

According to Jaspers (2010), by attending to microscopic phenomena of language use, one is able to keep a finger on the pulse of social tendencies prevalent in society regarding language. It means that studying how people use language provides useful insights into language and its relation with society. Unlike Chomskyan linguistics, which 


\section{F. Ahmad and G. Widén}

limits the concept of language to linguistic grammar and structure, sociolinguistics adopts a social view of language and insists on studying language in social interaction with a particular focus on language use (Lønsmann, 2011). A plethora of research has been done on how language-use practices at the individual level facilitate and constrain individuals' behaviour and actions. We see language use as a perspective that offers a specific theoretical and methodological mindset toward the study of language, particularly in terms of its relation to other factors - in our case, knowledge sharing.

Most previous studies on language and knowledge sharing acknowledge the relationship between these two factors. However, the relationship is usually established in a correlative style in which language in general is associated with some aspect of knowledge sharing, followed by some generic explanation. Quite often such studies fall short in their explanation of this influential relationship in objective terms. Instead of describing the linguistic practices that condition this effect on knowledge sharing, differences in cultures and communication styles are brought into the discussion (e.g., Klitmøller and Lauring, 2013; Peltokorpi, 2006). According to Welch and Welch (2008), when we discuss the relationship between language and knowledge sharing, it would be a useful strategy to respect language as a separate factor and try to focus specifically on language-related issues rather than mixing them up with other social factors such as culture. Such mixing makes it difficult to discern the effects of language from other relevant factors on knowledge sharing and consequently offers fewer insights into the development of knowledge-sharing strategies aiming to address the issue of language diversity in multilingual organisations (for further discussion, see Welch and Welch, 2008). This is where focusing on language use could prove to be a handy strategy. The focus on language use directs our attention to specific language practices that are important to understanding the ways language interacts with a phenomenon of interest (Jaspers, 2010). It provides an opportunity to anchor the explanation of the relationship between language and knowledge sharing in micro foundations, which ultimately means theorising the influence of language on knowledge sharing in terms of language behaviour rather than other social characteristics of individuals.

We introduce two types of language practices under the language-use perspective from sociolinguistics. Code switching and convergence have two characteristics in common that make them important and relevant for studying language use and knowledge-sharing relationships in multilingual contexts. First, these language practices, in one form or another, are widely prevalent in our daily social interactions and are known for their consequences for social engagements (Wardhaugh, 2010). Because a considerable amount of knowledge sharing takes place verbally (Peltokorpi, 2006), it is important to include these practices in our analysis of knowledge sharing in multilingual organisations. Second, and this is also related to the first one, the breadth and depth of these conceptual insights are enormous. Researchers interested in language have been assiduous in exploring the complexities of motivation and the form and consequences of code switching and convergence in different contexts and settings, including multilingual ones. A rather remarkable and sophisticated theoretical base offers knowledge-sharing researchers the opportunity to invoke these concepts in different forms and contexts in relation to knowledge sharing. In addition, it helps us in focusing on the main relevant issues rather than expending effort on secondary concerns. For example, while studying knowledge sharing in multilingual organisations, instead of finding out what causes code switching, which may have implications for knowledge sharing, we can invest our efforts in analysing the actual consequences of code switching for knowledge sharing. For 


\section{Knowledge sharing and language diversity in organisations}

motivation, we may look to previous research by sociolinguists that clarifies the motivational impetus for code switching. In practical terms, a good amount of research shedding light on different aspects of these concepts may provide useful insights into the development of potent knowledge-management strategies in multilingual organisations. It is important to attend to the theoretical base of a concept when interdisciplinary import is intended (Repko, 2012).

\section{Knowledge sharing and language use (switching and convergence)}

Knowledge sharing is a multi-faceted concept, and it has been approached from different perspectives and at different levels. A comprehensive review is not possible in this paper but we will outline here how we perceive this concept. According to Cummings (2003), there are two major aspects of knowledge sharing: "what facilitates or inhibits knowledge sharing" and the "actual act of knowledge sharing."

The first aspect is more general. It is concerned with factors that influence the opportunities for and possibility of knowledge sharing in an organisation. We call these knowledge-sharing opportunities in an organisation as knowledge-sharing potential. Previous research has identified a number of organisational and individual level factors that influence knowledge-sharing potential (e.g. Evans et al., 2012; Ipe, 2003; Wang and Noe, 2010). Knowledge-sharing potential is highly dependent on social interactions and personal networks in an organisation. When employees formally or informally engage with each other, they develop transactive memory (who knows what) and trust (Akgün et al., 2005). Moreover, social interactions at work also help in building social networks that, according to Hansen et al. (2005), are the major platform for knowledge sharing in organisations. In this paper, knowledge-sharing potential is mainly seen in the context of social interactions and networking at the workplace. This is in line with the personalisation perspective of knowledge management, which posits that the more social interaction there is between employees, the more likely they will share knowledge with each other (Noorderhaven and Harzing, 2009). In this paper, we will try to explore how code switching can influence knowledge-sharing potential in multilingual organisations with a specific focus on social interactions between employees.

The second aspect is more specific. It is concerned with what happens in the process of knowledge sharing between individuals (e.g. Bechky, 2003; Renzl, 2007; Sunaoshi et al., 2005). The process of knowledge sharing, particularly of a tacit kind, involves discussions and dialogue that facilitate sense-making and develop mutual understanding, for example, during a problem-solving episode. In this paper these knowledge-sharing discussions, also known as knowledge dialogues (Eppler, 2007), are called knowledgesharing interactions. We will discuss how convergence can influence knowledge-sharing interactions with a particular focus on communication and conversational aspects of such interactions.

Before turning to the main discussion, it is important to mention that code switching and convergence and their relationship with knowledge sharing are all seen in the context of multilingualism in this paper. This means that when we talk about switching, the focus would be on switching between languages in multilingual situations rather than switching between languages in monolingual situations. Similarly, language convergence in knowledge-sharing interactions would be taken in the context of convergence between participants who are multilingual, not monolingual. 


\section{F. Ahmad and G. Widén}

\subsection{Code switching and knowledge-sharing potential}

Code switching, also known as language mixing, is one of the most important concepts in sociolinguistics (Wardhaugh, 2010). Nunan and Carter briefly define code switching as "a phenomenon of switching from one language to another in the same discourse" (2001, p.275). Bilinguals and multilinguals are known for shifting between languages during discourse. They may start with one language and shift to another during the discourse. The magnitude of the shift can vary. Individuals may shift from language $\mathrm{X}$ to $\mathrm{Y}$ and continue their discussion in $\mathrm{Y}$ for some time, or they may shift to $\mathrm{Y}$ every now and then. It is important to recognise that code switching is not a random behaviour; instead it is a well-governed linguistic strategy used to convey linguistic and social information (Ritchie and Bhatia, 2013; Wei, 2013).

One of the main functions of adopting a common corporate language is to provide a medium of interaction that insures the quality of communication between linguistically diverse individuals (Marschan-Piekkari et al., 1999). Moreover, this also serves to neutralise possible language conflicts (Fredriksson et al., 2006). In the absence of any clear language policy, some language communities may engage in promoting their own language interests, leading to open frictions regarding language choice for communication, which may promote a hostile culture. In this scenario, it seems that the most ideal language practice for an organisation under its corporate language policy would be non-switching, which means the corporate language is used consistently within the organisation regardless of the topic, audience, and setting. Consistent use of the corporate language and not shifting to any other language in multilingual situations would provide an equal opportunity for all to participate. It would ensure that day-to-day conversations both in formal and informal contexts are in a common language. This would be helpful in the organisational cause of building a culture where social interactions between employees are not distorted by linguistic differences. Although this is an ideal situation, it is far from reality. First, the use of the common corporate language does not eradicate cultural and linguistic differences, and there is always room for conflicts and power games regarding language use. Second, corporate language policies are not always adhered to.

Organisations with an absence of any kind of code switching by multilingual employees would be an extremely rare case, one likely to occasion comment. A number of social and psychological factors may motivate individuals to shift between languages in a multilingual environment (Ritchie and Bhatia, 2013). Sometimes a speaker shifts to another language during the discourse because of her lack of proficiency in the second language. Lack of ability to find the right word or construct a sentence according to the thought may trigger the switch to another language. However, this is not the only reason for code switching. Speakers may shift between languages in order to show solidarity or distance to a language group, to establish identity, and assert power (Wei, 2013). In this case code switching is used as a "form of political expression" (Wardhaugh, 2010, p.101). Code switching is also used as a strategy to mask information, for example, for giving negative comments (Ritchie and Bhatia, 2013) or to conceal information from others (Emeka-Nwobia, 2014). Code switching is not always intentional. In societies where multilingualism is the norm, code switching quite often emerges as a subconscious linguistic practice that speakers themselves do not pay much attention to (Myers-Scotton, 1993). In short, code switching is a reality of many multilingual environments, including workplaces (Jan, 2003). In multilingual organisations with a corporate language as a 


\section{Knowledge sharing and language diversity in organisations}

common language for interaction, code switching can be of mainly two types: switching to the corporate language and switching away from the corporate language.

We specifically focus on code switching to and away from the corporate language, and we do this for two reasons. First, the corporate language is an important language in organisation because of its official status that makes it one of the most widely used languages in the organisation. Second, in organisational context, code switching can also occur between any other two languages, particularly in case of trilingual employees. This adds into the complexity of the concept. It is not possible to cover all such possibilities in this single paper, so we will focus only on code switching to and away from the corporate language. In the following discussion, we shed light on code switching in the context of multilingual organisation and what implications this kind of language use has for the knowledge-sharing potential.

\subsubsection{Switching to the corporate language and knowledge-sharing potential}

In organisations, informal social interactions between employees are considered as a breeding ground for social networks (Reychav and Te'eni, 2009; Tange and Lauring, 2009). These interactions provide a platform where people can talk about their work routines, share complaints and opinions, and discover the interests and skills of their fellow colleagues. Such social interactions build informal links and transactive memory systems that play an integral role in creating knowledge-sharing possibilities (Akgün et al., 2005). Probably this is why many organisations nowadays try to promote social interaction between employees by building common activity rooms and designing workplaces that create opportunities for small talk (Bakke, 2007). However, the success of such efforts is to a great extent influenced by the linguistic behaviour of the employees in the multilingual organisations, because social interactions, particularly informal ones, also constitute the domain where employees are likely to use their own language for conversation (e.g. Tange and Lauring, 2009; Remennick, 2005). In this scenario, switching to the common corporate language becomes important for the knowledgesharing potential, particularly when the situation shifts from monolingual to multilingual. A multilingual situation is characterised by the presence of an audience with linguistically diverse backgrounds. This audience does not mean only the addressee(s) but may also include the people around the interlocutors who may be listening to the conversation; these people are called auditors, overhearers and eavesdroppers by Bell (2001). Consider a situation where a group of Italians having a discussion in Italian shift their language to English when they notice that their English-speaking colleague has just approached the group. This shift toward the corporate language is a language practice that, if deployed quite often in social interactions, would generally have positive consequences for the knowledge-sharing potential. Shifting to the common corporate language in acknowledgment of a multilingual audience, who may not be interlocutors, would contribute positively in building a pleasant environment in a multilingual organisation. It allows the members of different linguistic groups to be part of the discussion and networking regardless of their linguistic differences. It promotes an open culture where access to information and discussions does not run along linguistic boundaries. Code switching to the corporate language would be helpful in maintaining and even developing cross-linguistic social interaction, which is a basic building block of the knowledge-sharing potential in multilingual organisations. 


\section{F. Ahmad and G. Widén}

\subsubsection{Switching away from corporate language and knowledge-sharing potential}

One of the most interesting and, in our view, very influential type of code switching for knowledge sharing is switching away from the corporate language, particularly in multilingual situations. At first it may seem quite unlikely that any participant in the discourse would switch away from corporate language in a multilingual situation. Such a language practice apparently violates the corporate language policy as well as the cooperative philosophy of organisations, which are social institutions where cooperation is an expected norm. However, some studies in closely related areas have noticed that switching away from common corporate language is quite a common practice in multilingual organisations (e.g. Hinds et al., 2014). This may be because many of the social principles, such as culture, power, identity, solidarity, that govern social interaction in our daily life outside work are also in action in social interactions within organisations. For the same reasons that justify the positive relationship between switching to the corporate language and the knowledge-sharing potential, switching away would have negative impact on the knowledge-sharing potential. For example, in the above-mentioned example if Italians greet the incoming English speaker and switch back to their own language, it would clearly signal that they are more interested in carrying on their conversation in their own language rather than ensuring the participation of the nonnative speaker. If such behaviour is common among different language groups, it can isolate them from each other. Moreover, it might also create language clusters (languagebased grouping). Such clusters develop due to the tendency of employees to interact more with their own speech community (Ahmad and Widén, 2015). Language clusters act as knowledge networks and negatively influence knowledge sharing between employees with diverse linguistic backgrounds (Tange and Lauring, 2009).

It is not always in informal social interactions that switching away from the corporate language is possible; indeed, this also happens on formal occasions. One of the respondents, in a study on language diversity and social interaction in a Danish company by Tange and Lauring (2009), outlines the scenario of a meeting that we may consider an example of code switching away from the corporate language. He describes a meeting where participants were from different linguistic backgrounds (English, Danish and Swedish). After some time both Danes and Swedes were busy talking in Swedish and Danish, and they did not switch back until they were asked to do so. Code switching away from the corporate language could occur for many reasons. The most important of them is language proficiency. Employees with limited linguistic competency in the corporate language are more likely to switch away from the corporate language, particularly when they are unable to find the right words (Hinds et al., 2014). Another reason could be power dynamics: even though the employees do have the required proficiency, they may still shift from the corporate language to exert power or show their language identity (Lønsmann, 2011).

Generally, switching away from the corporate language is most likely to attract the criticism and anger of participants who are precluded from participating in discussion. Many sociolinguistics studies underline the negative attitude of the people toward code switching (e.g. Berthele, 2012). Not only the audience but also speakers who themselves switch between languages are sometimes critical of their own code-switching practices when they are brought to their attention (Dewaele and Wei, 2014). Particularly in organisational contexts, speakers are more likely to react negatively to such switching, 


\section{Knowledge sharing and language diversity in organisations}

because in multilingual environments usually the corporate language is the unmarked code for communication. It is expected and accepted; however, when people shift to the marked code (e.g. native language) in the middle of the discourse, this generates usually negative reaction from interlocutors. In a study on multilingual teams, Hinds et al. (2014) found that Americans were very critical of Germans who were in the habit of using German for short intervals in project meetings. It made the Americans feel like outsiders and suspect they were being talked about. Such a switching away from corporate language in formal multilingual environments is harmful to the knowledge-sharing potential in two ways. First, it marginalises the non-speakers of the language and excludes them from the discussion in progress. Switching away from the corporate language creates blank spots in communication for the multilingual audience. These blank spots could leave the audience without information that may be critical for them and hence limit learning opportunities and collaboration. Second, such code switching generates mistrust and prompts negative emotions such as anger and distress, which has consequences for the knowledge-sharing potential in the long run. The audience may believe that switching away from the common language is an intentional strategy to hide some important information or to pass on negative comments about them. It may perpetuate or even escalate resentment and mistrust among employees in the organisation. Based on our discussion so far, we can derive the following propositions.

Proposition 1: Code switching is likely to influence the knowledge-sharing potential in a multilingual organisation.

Proposition 2: Code switching to the corporate language in multilingual situations is likely to have positive effects on the knowledge-sharing potential between linguistically diverse employees in a multilingual organisation.

Proposition 3: Code switching away from the corporate language in multilingual situations is likely to have negative effects on the knowledge-sharing potential between linguistically diverse employees in a multilingual organisation.

Before we move on, it is important to elaborate that proposition two holds one very important assumption, namely that there is no ongoing linguistic conflict between language groups. If a language group has negative attitude toward the corporate language or a language community (see Vaara et al., 2005), then shifting to the corporate language in a multilingual audience may not have any considerable positive effect on the knowledge-sharing potential between linguistically diverse employees. Then their switching to the corporate language is more likely to be to accommodate the multilingual audience in the short term, for example, for asking a specific question or just to be polite. But in the long term, they may intentionally abstain from engaging in knowledge and information exchange with linguistically diverse employees due to the conflict and language power dynamics (Lauring and Bjerregaard, 2007). Since knowledge is power in organisations, therefore in conflict-ridden environments, employees do not exchange their knowledge with each other (Chen, 2011).

\subsection{Convergence and knowledge-sharing interaction}

Quite often we have noticed that salesmen tune their language style to suit the customer. A salesman at an electronic store briefing an old customer about the features of a mobile phone may adopt a totally different language style compared the one he uses for a young 


\section{F. Ahmad and G. Widén}

customer. This variation in language reflects a strategic use of language in relation to the interlocutor. This phenomenon is well known as language convergence in sociolinguistics and social psychology and is an integral part of the communication accommodation theory. Language convergence refers to the ways in which interlocutors adjust or change their linguistic behaviour to become more similar to an interacting partner. The change may be in terms of accent, speaking rate, intensity, pause frequency, and utterance length (Pardo et al., 2012). In other words, convergence can be seen as "speakers' attempts to attune positively along major sociolinguistic dimensions to the characteristics which they believe belong to the speaker receiving their message" (Gallois and Callan, 1988, p.271).

Language convergence is usually perceived positively by others. Individuals are rated quite highly by interlocutors on a number of social dimensions such as perceived supportiveness, intelligibility, cooperativeness, and trust because of their language convergence (Giles and Coupland, 1991). Moreover, convergence by bilinguals in their common language enhances the quality of communication (Cogo, 2009). It may be suggested that language convergence is likely to have a positive influence on knowledgesharing interactions. Positive attitude, trust, perceived supportiveness, and high intelligibility are all such features that usually contribute highly to the success of knowledge-sharing interactions (Eppler, 2007; Evans et al., 2012). Knowledge sharing is more than an act of message transmission. It is a collaborative and sense-making process in which the sender attempts to translate her insights, hunches, experiences, and context into language understandable to the receiver. To achieve this purpose efficiently, a myriad of "didactic tricks" and "speech acts" are required (Eppler, 2007), and convergence is one such linguistic strategy useful for building positive interaction. However, language convergence is not always mutual. Sometimes interlocutors do not reciprocate convergence at all, and in other cases they may converge only partially (Giles and Coupland, 1991). This underlines that there is variation in terms of language convergence between interlocutors, and this variation is influenced by the goals of the interlocutors. According to the previous literature, there are two main functions of language convergence. People converge in order to secure the approval or the support of the others (Giles and Coupland, 1991). A quite common example is the communication style of the politicians who sometimes adopt folksy language in their speech with common people (Wardhaugh, 2010). A second function of convergence is communication proficiency (Giles and Powesland, 1997). While taking into account the requirements of the listeners, speakers modify their speech to facilitate comprehension and clarity in communication (Gallois et al., 2005).

In the following discussion, we will analyse language-convergence behaviour in knowledge-sharing interactions in multilingual environments while keeping the variation aspect in focus. We frame our discussion in the context of two functions of language convergence.

\subsubsection{Convergence for efficiency and knowledge-sharing interaction}

Initiation of language convergence depends to a great extent on the desires and goals of the interlocutors. Two strangers having a short conversation at a train station are less likely to converge than individuals trying to solve a complicated problem in an organisation. There is clearly a difference in the goals of both conversations. Language convergence reduces the difference between interlocutors at both verbal and the 


\section{Knowledge sharing and language diversity in organisations}

psychological levels (Gallois et al., 2005). Therefore, in certain communication situations characterised by shared goals and contextual conditions, language convergence would be the common case (Giles and Coupland, 1991; Giles and Powesland, 1997). Knowledge sharing is likely to be one of these situations, because successful knowledge transfer is the ultimate purpose of both sender and receiver (Cummings, 2003). After all, this is the very reason that a knowledge-sharing interaction has been initiated by two parties. Knowledge exchange at the individual level is usually more of a voluntary and independent effort guided by personal links of employees and a sense of community (Ipe, 2003). In this scenario, knowledge-exchanging colleagues are more likely to engage in cooperative tactics that would allow them to secure their goal of successful transfer, and at the speech level language convergence is likely be one such tactic, particularly in multilingual situations. It is quite likely that most knowledge-sharing interactions at the individual level would involve at least some form of language convergence.

Although the goal of interaction provides a useful clue to the deployment of a convergence strategy, to what extent interlocutors will converge depends to a great extent on their perception of the similarities and differences between themselves (Coupland, 2001; Coupland et al., 2001). Convergence is influenced by how individuals relate to each other (Wardhaugh, 2010). The greater the perceived differentiation and the greater the need to achieve communication efficiency, the greater the convergence between interlocutors would be. This means that individuals with different linguistic backgrounds would converge more than individuals with similar linguistic backgrounds in their knowledge-sharing interactions. This is because the perception of a differential between interlocutors with dissimilar linguistic backgrounds is usually greater than between interlocutors with similar linguistic backgrounds. In multilingual organisations where people with different linguistic backgrounds are involved, the sense of dissimilarity in social interaction is usually high (Ahmad and Widén, 2015). This becomes more evident when interaction involves native and non-native speakers or when linguistic dissimilarity coincides with cultural dissimilarity. Language dissimilarity is known to increase or decrease the perception of cognitive and psychological disparity. For example, a Spanish manager would have a very different perception and expectation of her interaction in a meeting with a Chinese as compared to a Portuguese, mostly because of the difference in their languages (Schomaker and Zaheer, 2014). As mentioned above, knowledge sharing is a cooperative venture motivated by the desire to successfully accomplish the task at hand. Needless to say, for the exchange of knowledge, particularly tacit knowledge, efficient and effective interaction is of the utmost importance (Eppler, 2007; Fernie et al., 2003). In this scenario where, on the one hand, the psychological distance between interlocutors is great due to language dissimilarity and, on the other hand, effective communication is critical, linguistically diverse interlocutors would engage in a high level of speech convergence in their knowledge-sharing interactions. While noticing and evaluating the difference in each other's language capability, linguistically diverse interlocutors may engage in different convergence strategies to modify the complexity of the speech such as speaking slowly, using simple vocabulary, neutralising accent, eschewing culturally sensitive words and slang, and showing politeness (selfdepreciation with Japanese) (see Giles and Coupland, 1991).

On the other hand, in the case of knowledge-sharing interaction between natives of the language in use, the convergence situation is likely to be different. They may converge, but their convergence is likely to be less extensive, because their perception of differentiation is less. When people speak with someone from their speech community, 


\section{F. Ahmad and G. Widén}

they usually also assume common understandings, which influences the way they adjust their speech (Bechky, 2003). Language similarity increases cognitive closeness and reduces the physic distance and perception of differentiation (Makela et al., 2007; Selmer and Lauring, 2015; West and Graham, 2004), so it is likely to reduce the intensity of convergence in knowledge-sharing interactions. Since convergence in cooperative interaction is the function of perceived differentiation and since language increases or decreases this differentiation, variance in language associations would lead to variance in language convergence in knowledge-sharing interactions.

\subsubsection{Convergence for approval and knowledge-sharing interaction}

Language convergence is quite often in evidence when there is asymmetry in power between interlocutors. For example, an employee would converge more than a supervisor in their information communication, a witness is likely to converge more than the interrogator, and an interviewee would be converging more than the interviewer in a job interview (Giles and Coupland, 1991). In short, it means that in hierarchal relationships an interlocutor who is low in the hierarchy is more likely to converge than the interlocutor who is high in the hierarchy. In such a relationship there is clear benefit for the one party to converge, for example the interviewee may get a job, the witness may make the interrogator believe her story, and the employee may secure the support and approval of the supervisor in the organisation. The convergence for approval principle would be applicable in knowledge-sharing interaction between multilinguals. It is based on the well-known postulate that language is a source of power, and it promotes asymmetrical relationship between language communities in the organisation (Ahmad and Widén, 2015; Vaara et al., 2005).

In a multilingual organisation, languages represent different language communities in the organisation. Some languages and their communities are considered more powerful than others in terms of the official hierarchy and access to information and knowledge. Proficiency in a certain language may allow access to an informal network of information that otherwise may remain hidden (Welch and Welch, 2008). It is quite often the case that majority-language speakers in the organisation are dominant over other language communities in the organisation. This is particularly the case when the corporate language and the local language of the country is the one and the same. However, in some other scenarios even the minority languages can be dominant over the others. For example, in a case study on Danish expatriates in a Saudi Arabian subsidiary of a Danish company, Lauring and Bjerregaard (2007) found that the top management of the subsidiary was mainly composed of Danish managers. It was a purposeful strategy that allowed them to contain the information by using Danish only. In this situation, Danish appeared to be the powerful language in terms of access to valuable information as compared to Arabic, which was the majority language. It is not easy to pinpoint the power status of a language (community), but previous research suggests a number of factors that provide useful clues to determining at least the language(s) that are at the top of the power hierarchy, such as the composition of organisational managerial force (Mcall, 2003; Lauring and Bjerregaard, 2007), the language of the local country (Amelina, 2010) and the organisational language policy (Thomas, 2008). All in all, there is almost always language a hierarchy in multilingual organisation in which some 


\section{Knowledge sharing and language diversity in organisations}

language groups enjoy access to critical information due to their ability to speak a certain language. As a result, there is asymmetry in terms of power (access to information) across different language groups.

A language group that has access to more resources and information may become more attractive to other language groups. In such a scenario when there is power distance between two language groups, members from the low-hierarchy language group are more likely to converge in their knowledge-sharing interaction with members from the highhierarchy language group. There is a direct benefit for low-hierarchy language group members to secure approval and support of the high-hierarchy language group members. Securing approval of high-hierarchy language group members would pave the way for the possibility of invoking the principle of reciprocity in the future. Individuals with a converging language in discourse are more likely to have their volunteered assistance requests entertained (Buller and Aune, 1988). In this way they may secure access to their information network, which may contain useful information residing beyond the direct access of the different-language speaker. In this scenario, successful knowledge-sharing interaction is in the best interest of the low-hierarchy language group members when they engage with high-hierarchy language group members, simply because they have more to gain with the success of knowledge-sharing interaction. Therefore, they may invest more energy in general in this process, whereas at the speech level language convergence is likely to be employed as a tactic to enhance the quality of knowledge-sharing interaction and hence improve the chances of knowledge-transfer success. It may highlight them as professional and competent individuals in the organisation, characteristics highly valuable in securing support and approval. Besides the purpose of making knowledgesharing interaction a success, there is another reason that may lead to a high level of convergence by low-hierarchy language groups. The process of knowledge sharing, to some extent, manifests participants' characteristics. It demonstrates about the abilities and potential of the participants involved in carrying out a successful transfer interaction. It shows the knowledge reservoir of the participants as well as their capacity to disseminate that knowledge properly. In this scenario, convergence in knowledge-sharing interaction can also be used as a useful weapon for impression management (assertive self-presentation), that is, to portray a competent persona. This positive impression provides leverage in future knowledge-sharing transactions, because experience of one's past dealings with an individual influences subsequent endeavours between them. In short, a power distance between two language groups would lead to high level of language convergence from the language group low in the hierarchy in knowledgesharing interactions. Based on our discussion so far we may derive the following propositions.

Proposition 4: Convergence will have positive effects on knowledge-sharing interaction.

Proposition 5: Convergence is likely to be more in evidence in knowledge-sharing interaction between non-natives (one or more) than native speakers of the language in use.

Proposition 6: In knowledge-sharing interaction between minority and majority (in terms of power) language-group members, minority language participants are likely to converge more than the majority-language participants. 


\section{Future research directions: the role of language practices in knowledge sharing}

In the above discussion, we have attempted to establish a relationship between two types of language practices and knowledge sharing. Given the vast scope of knowledge sharing and language use (code switching and convergence), this first attempt, according to our knowledge, to analyse the relationship between these two is quite selective and focused. Practically speaking, it is beyond the reach of this paper to touch upon all the aspects of code switching and convergence in relation to knowledge sharing, and therefore the aim was to look at these language-use concepts in their general nature. By bringing together insights generated in empirical and theoretical studies in both fields, we have put forward some propositions. A proposition-based framework is useful in the introduction of insights emanating from a different discipline and in mapping the potential relationship between constructs under discussion (McDonald and Tang, 2014). Although some of the propositions are more general than others, they direct our attention to some specific topics and areas of research. Empirical studies are needed to verify the logical arguments presented in the propositions. Even the rejection of the propositions in empirical studies would provide very useful insights for our research on language practices and knowledge sharing. For example, if code switching does not influence knowledge sharing then what are the reasons, and how does this add into our existing beliefs about the influence of language on knowledge sharing. As has already been mentioned, we do not attempt to cover all the aspects of relationships between code switching, convergence, and knowledge sharing. It means, in addition to what we have discussed so far in this paper, there is ample opportunity for further research. Some of these possibilities are discussed below.

In this paper, we have discussed code switching as a language practice only in the context of knowledge-sharing potential. However, another interesting theme is the exploration of code switching behaviour within knowledge-sharing interactions, for example, in project meetings. Some fields and topics of discussion are greatly influenced by certain languages; for example, in technology-related professions there are many expressions, concepts and terminologies that are usually expressed in English rather than in local languages. In this scenario, it would be interesting to explore how code switching, as a practice, is used as a resource in different types of knowledge-sharing interactions and what consequences this has for the knowledge-sharing process. Similarly, code switching in written material such as emails, internal news bulletins and documents may also be interesting to look at in relation to knowledge sharing.

Language convergence has been discussed here in the context of knowledge-sharing interaction. However, it may have a very influential relationship with knowledge-sharing potential as well. Frequent convergence in social interactions at work may bring some people closer, creating strong relationships, which can increase the potential for knowledge sharing in the future. This proposition goes against the traditional perception of language as divisive and segregating language communities in an organisation. In some cultures, communication is more receiver-centred than sender-oriented (Yum, 1988), which means people from some cultures are more likely to converge than others. It provides an opportunity to look at the language-convergence dynamics in knowledge transfer across borders. Although we have focused here only on the positive aspects of the convergence in its relationship with knowledge sharing, previous research suggests that convergence may also have negative implications for social interaction. In an effort 


\section{Knowledge sharing and language diversity in organisations}

to conceptualise and tune the linguistic discourse, the content of the discourse may be compromised. It is well known that alternation in knowledge content during knowledge transfer usually influences the quality of the knowledge transfer (Higgins, 1999). This means future research should also explore the negative influence of language convergence on knowledge sharing (interaction).

What types of interventions are required to manipulate code switching and convergence in order to improve knowledge sharing is also an interesting and important area of inquiry. There is little about how these language practices can be regulated or manipulated. In this regard, knowledge-sharing researchers have to put some effort into finding strategies that can align code switching and convergence with the interests of organisational knowledge management. At a minimum, organisations should attempt to systematically bring these linguistic practices to the greater attention of employees in order to motivate them to reflect on their language behaviour and its consequences for social interaction in general and knowledge sharing in particular.

\subsection{Methodological suggestions}

In methodological terms, one of the most important questions for knowledge-sharing researchers is how to collect data regarding the language practices discussed in this paper. A number of data-collection methods such as questionnaires, interviews and observations have been used for this purpose in sociolinguistics and related disciplines (Wei and Moyer, 2009). Nevertheless, conversation recordings stand out as an essential method to study language practices in general and code switching and language convergence in particular (Clemente, 2008, p.177). Nonetheless, we do not find the application of this method in the knowledge-sharing literature, which relies heavily on the self-reports of respondents generated through interviews and questionnaires to study the influence of the language. For example, in a study on language diversity and knowledge sharing, researchers of a multilingual university in Denmark were asked to report their language practices in a questionnaire (Lauring and Selmer, 2011). Based on these self-reports, language practices were correlated with knowledge-sharing behaviour and performance. One of the problems with the self-reported language practices is the possibility that answers may reflect attitudes toward language rather than actual language behaviour. When a certain language choice or behaviour is commonly considered to be negative, respondents are likely to underreport that behaviour and vice-versa (Nortier, 2008, p.37). For example, Nortier (2008) reports an incidence during an interview in which a Dutch Moroccan woman was asked about the use of the Arabic at home. She strongly denied that she would ever use Arabic at home while living in the Netherlands, where Arabic does not enjoy any prestige. However, at that particular moment her daughter approached and she quickly shifted to Arabic in conversation with her daughter, forgetting what she had reported before. This may also be the case in organisations with a one-language policy in place, and respondents may underreport or even hide their shift away from corporate language practices. Moreover, language behaviour is sometimes subconscious, which means respondents may experience problems in recalling their actual language practices, as has been noted in Blom and Gumperz (1972).

Quite often natural conversation recordings are combined with questionnaires and interviews, depending upon the purpose of the study. Audio recording of natural conversations is a well-known and rather reliable method for data collection regarding language practices. Based on the potential benefits associated with natural conversation 


\section{F. Ahmad and G. Widén}

recordings, we suggest that knowledge-sharing researchers should also employ this method for studying the dynamic relationship between language practices and knowledge sharing.

Some care is warranted in generalisations of results implied from recorded data. Conversation tapes, which consist of certain communicative events, may not be representative of the general language practices prevalent in the organisations, and employees may have different types of language practices in different domains within organisations. One useful strategy to solve this issue would be to thematically categorise the communication episodes in the organisations, for example, meetings, lunch talk, project discussion, small talk in corridors etc. In this scenario, language practices appearing from a certain type of communication episode may be generalised to the relevant theme only. How to secure permission for accessing and recording natural workplace conversations is also an important issue that must be carefully considered beforehand. In this regard some useful suggestions that are particularly relevant to the workplace context are given by Holmes and Stubbe (2015).

In the above discussion, we suggested recording conversations as a useful datacollection technique for language practices in context of knowledge sharing. Now we will go on to discuss methodological possibilities in relation to our suggested propositions.

For code switching and knowledge-sharing potential, longitudinal ethnography would be a useful methodology. Longitudinal ethnography provides a useful framework to analyse the environment in its natural form over an extended period of time. Ethnographic study should combine recoded observations and interviews with sociometric questionnaires. A sociometric questionnaire provides a diagram of network connections in an organisation, thus reflecting the knowledge-sharing potential between different groups in an organisation (Ibarra, 1992). Sociometric questionnaires show how connections evolve over time and are a useful technique to anticipate the likely flow of knowledge within an organisation. Recorded observations should focus on codeswitching practices in day-to-day interactions, including informal discussions such as coffee talk. While seeing knowledge-sharing potential in terms of connections and understanding code-switching practices in social interactions, in-depth interviews should be conducted with appropriate candidates who have interesting profiles regarding their network connections and code-switching practices. These interviews should aim to highlight the possible connection between code switching and knowledge-sharing potential.

Ethnography would also be useful for the analysis of convergence and knowledgesharing interaction. It would require in-depth observation of knowledge-sharing interactions that should be observed in a specific context. For example, interactions during a technology transfer (Sunaoshi et al., 2005) and critical problem-solving in teams (Klitmøller and Lauring, 2013) could be categorised as knowledge-sharing interactions. If allowed, knowledge-sharing interactions should be recorded. A useful analytical method regarding convergence and knowledge-sharing interaction is conversation analysis, provided that recordings are available. Conversation analysis is conducted on interactions with the aim of finding certain recurring patterns of interactions. In the case of knowledge-sharing interactions, it could be useful in identifying not only convergence patterns in speech but also what leads to such patterns at the interactional level. Moreover, in-depth interviews can also be used with observations particularly to understand the experience of knowledge-sharing participants regarding convergence and 


\section{Knowledge sharing and language diversity in organisations}

whether such convergences enhance the quality of knowledge sharing. In our view, instead of having a strict definition of convergence, researchers should proceed with an open mind and should focus on all language variations that are meant to accommodate knowledge-sharing participants.

The above suggestions do not exhaust the list of possible ways to test the propositions presented in this paper. Even a quantitative research design can be used to test these propositions. However, a qualitative study or a mixed-method study seems to offer a better framework and explanations, particularly regarding language use.

\subsection{Limitations}

In this paper, we are studying knowledge sharing in a general sense. We do not contextualise knowledge sharing. Although this kind of perspective has been adopted in previous studies such as Ahmad and Widen (2015), Welch and Welch (2008), we believe more useful insights can be generated when knowledge sharing is contextualised, for example, in terms of type of knowledge, space (virtual, face to face), structure (teams, hierarchy) etc. A good example of such contextualising in knowledge sharing is a study by Sunaoshi et al. (2005), who investigated the technology transfer between Japanese expatriates and American production-floor employees in a car plant. Although such contextualisation presents the issue of the generalisability of findings due to the very specific context, it can be useful for developing deep insights and therefore is recommended for future research on language and knowledge sharing.

Another limitation concerns the complexity of the two sociolinguistic concepts presented in this paper. These two concepts are very dynamic and contain a plethora of views and complex interpretations that need to be touched upon for a better understanding of their relation with knowledge sharing. For example, in code switching, we focused only on code switching to and away from corporate language, although, as mentioned earlier, code switching can also happen in languages other than the corporate language in an organisation. In Finland, which has a bilingual population, code switching between Finnish and Swedish is quite common at workplaces with English as corporate language. However, this was a limitation that we had to accept due to the limited space and the novelty of the topic at hand. Further research can pursue more in-depth analyses of these concepts in relation to knowledge sharing.

In the recent years, the concept of code switching has been questioned. There has been some criticism in viewing language as separate entities and the idea of dual monolingualism has been challenged. It is suggested that individuals with knowledge of multiple languages use it as a single resource in social interactions (Cruz-Ferreira, 2006). These multiple languages are not different linguistic repertoires, and therefore should not be seen and treated as distinct repertoires, as is usually done in code-switching research. This thinking has led to the introduction of new concepts such as translanguaging (García and Wei, 2013), polylingual languaging (Jørgensen, 2008), and code meshing (Young and Martinez, 2011) that are relevant to multilingual environments but do not create boundaries between languages. We strongly recommend that future research on language and knowledge sharing should also look into this perspective on language. This new view underlines multilingualism as a resource and hence has the potential to offer useful insights for knowledge-sharing research that has largely seen multilingualism as a problem. 


\section{F. Ahmad and G. Widén}

\subsection{Contributions}

Language is a broad phenomenon that can be studied in different ways. Previous research has mainly studied the relationship between language and knowledge sharing in the context of diversity. For example, this kind of research has underlined how language diversity can lead to social isolation that negatively influences cross linguistic knowledge sharing (Ahmad and Widen, 2015; Makela et al., 2007). In this paper we have tried to move from more general to more specific role of language in knowledge sharing. We do this by focusing on language use and its relationship with knowledge sharing. It is now well established that language directly or indirectly has consequences for knowledge sharing in multilingual organisations. However, to further our understanding of this relationship, we need to theorise in terms of individuals' actions, that is, how language is used in a multilingual organisation. This is in accordance with views of social scientists who consider individuals' actions a unit of analysis for building theoretical cause-effect explanations (Coleman, 1990; Elster, 2007).

One of the problems in the recent literature on knowledge sharing is how to study the influence of language on knowledge sharing. Schomaker and Zaheer (2014) propose that to understand the real influence of language on knowledge sharing, we should focus on structural aspects of language (such as the structural similarity between the languages of knowledge-sharing participants), as contrasted with its functional aspects (use of language by employees). According to them, structural aspects of language are not influenced by other social factors such as culture, and therefore let us see the influence of language on knowledge sharing in pure linguistic terms. Our paper contributes to this debate by presenting a potential relationship between language practices and knowledge sharing, and hence suggesting that functional aspects are equally important. Moreover, it also shows that by focusing on language practices, we will not only reveal the real nature of language, which is social, but can also theorise the influence of language on knowledge sharing while maintaining a focus on language only.

Another important contribution of this study is the introduction of sociolinguistic concepts in knowledge-sharing literature, which has not been specifically attempted before. Knowledge management as a field has been quite welcoming to crossdisciplinary concepts. Many previous studies have consulted with other disciplines in their analysis of knowledge sharing in organisations. Wang and Noe (2010) report a list of cross-disciplinary theories and concepts that have been used in knowledge-sharing research. However, in the context of language and knowledge sharing, we do not find any considerable efforts that consult with linguistics. An exception is Ahmad and Widen (2015), who introduced a theory from linguistic anthropology to explain language clustering and its influence on knowledge sharing in multilingual organisations. Our present paper underlines the connection between sociolinguistics and knowledge-sharing research, which is quite natural because of the theoretical enrichment and understanding that sociolinguistics offers regarding language in society. Such an interdisciplinary dialogue will be helpful in developing theory and better understanding the relationship between language and knowledge sharing in organisations. 


\section{Knowledge sharing and language diversity in organisations}

\section{Conclusion}

In this paper, we have attempted to introduce a new perspective on language in the context of knowledge sharing in multilingual workplaces. The focus on language use has been suggested for conceptualising the influence of language on knowledge sharing. Code switching and convergence are two important language practices that are common in social interactions. Building on the previous literature on language in sociolinguistics and knowledge sharing in knowledge management, we have attempted to show how code switching and convergence may influence knowledge sharing in multilingual organisations. In this effort, we have introduced some propositions that summarise the conceptual links and direct our attention to potential areas of inquiry for further research. In addition to offering a strong theoretical base, code switching and convergence provide very useful and objective constructs for analysing the relationship of language with knowledge sharing. This creates possibilities for a comparison of the results generated in knowledge-sharing studies investigating the influence of language. Research on language and knowledge sharing in multilingual contexts is still in its initial phase and hence is very limited. This is apparent in a recent review of knowledge-sharing literature where only a fleeting reference was made to language as an influential factor (Wang and Noe, 2010). In this regard our current effort of enriching the concept of language with sociolinguistic insights will hopefully be a useful addition in knowledge-sharing research in multilingual organisations. Understanding the influence of code switching and convergence on knowledge sharing is one step toward a better understanding of knowledge sharing as a whole in multilingual organisations.

\section{References}

Ahmad, F. and Widen, G. (2015) 'Language clustering and knowledge sharing in multilingual organizations: a social perspective on language', Journal of Information Science, Vol. 41, No. 4, pp.430-443.

Akgün, A.E., Byrne, J., Keskin, H., Lynn, G.S. and Imamoglu, S.Z. (2005) 'Knowledge networks in new product development projects: a transactive memory perspective', Information \& management, Vol. 42, No. 8, pp.1105-1120.

Amelina, M. (2010) 'Do other languages than English matter? International career development of highly-qualified professionals', in Meyer, B. and Apfelbaum, B. (Eds): Multilingualism at Work from Policies to Practices in Public, Medical and Business Settings, John Benjamins Pub. Co., Amsterdam Philadelphia, pp.235-252.

Bakke, J.W. (2007) The Nordic workplace design for knowledge work, Nordic Innovation Centre.

Bechky, B.A. (2003) 'Sharing meaning across occupational communities: the transformation of understanding on a production floor', Organization Science, Vol. 14, No. 3, pp.312-330.

Bell, A. (2001) 'Back in style: reworking audience design', in Eckert, P. and Rickford, J. (Eds): Style and Sociolinguistic Variation, Cambridge University Press, pp.139-169.

Berthele, R. (2012) 'The influence of code-mixing and speaker information on perception and assessment of foreign language proficiency: an experimental study', International Journal of Bilingualism, Vol. 16, No. 6, pp.1-14.

Blom, J.P. and Gumperz, J. (1972) 'Social meaning in linguistic structures: code switching in northern Norway', in Gumperz, J. and Hymes, D. (Eds): Directions in Sociolinguistics; the Ethnography of Communication, Holt, Rinehart and Winston, New York, pp.407-434. 


\section{F. Ahmad and G. Widén}

Bock, G., Zmud, R.W., Kim, Y. and Lee, J. (2005) 'Behavioral intention formation in knowledge sharing: examining the roles of extrinsic motivators, social-psychological forces, and organizational climate', MIS Quarterly, Vol. 29, No. 1, pp.87-111.

Buller, D.B. and Aune, R.K. (1988) 'The effects of vocalics and nonverbal sensitivity on compliance a speech accommodation theory explanation', Human Communication Research, Vol. 14, No. 3, pp.301-332.

Chen, Z. (2011) 'The interactive effects of relationship conflict, reward, and reputation on knowledge sharing', Social Behavior and Personality: An International Journal, Vol. 39, No. 10, pp.1387-1394.

Clemente, I. (2008) 'Recording audio and video', in Wei, L. and Moyer, M. (Eds): Research Methods in Bilingualism and Multilingualism, John Wiley \& Sons, pp.177-191.

Cogo, A. (2009) 'Accommodating differences in ELF conversation: a study of pragmatic strategies', in Mauranen, A. and Ranta, E. (Eds): English as a Lingua Franca: Studies and Findings, Cambridge Scholars, Newcastle, pp.254-273.

Coleman, J.S. (1990) Foundations of Social Theory, Belknap Harvard, Cambridge, MA.

Coupland, N. (2001) 'Language, situation and the relational self: theorizing dialect-style in sociolinguistics', in Eckert, P. and Rickford, J. (Eds): Style and Sociolinguistic Variation, Cambridge University Press, pp.185-210.

Coupland, N., Sarangi, S. and Candlin, C.N. (2001) Sociolinguistics and Social Theory, Longman, Harlow.

Cruz-Ferreira, M. (2006) Three is a Crowd? Acquiring Portuguese in a Trilingual Environment, Multilingual Matters.

Cummings, J. (2003) Knowledge sharing: A review of the literature, World Bank, Washington, DC.

Dewaele, J. and Wei, L. (2014) 'Attitudes towards code-switching among adult mono-and multilingual language users', Journal of Multilingual and Multicultural Development, Vol. 35, No. 3, pp.235-251.

Elster, J. (2007) Explaining Social Behavior: More Nuts and Bolts for the Social Sciences, Cambridge University Press, Cambridge.

Emeka-Nwobia, N.U. (2014) 'Code switching in igbo-english bilingual conversations', British Journal of English Linguistics, Vol. 2, No. 3, pp.1-6.

Eppler, M. (2007) 'Knowledge communication problems between experts and decision makers: an overview and classification', The Electronic Journal of Knowledge Management, Vol. 5, No. 3, pp.291-300.

Evans, M., Wensley, A. and Choo, C.W. (2012) 'How shared language and shared vision motivate effective knowledge sharing behavior', 13th European Conference on Knowledge Management - ECKM 2012 proceedings, Universidad Politécnica de Cartagena, Academic conferences international, Spain.

Fernie, S., Green, S.D., Weller, S.J. and Newcombe, R. (2003) 'Knowledge sharing: context, confusion and controversy', International Journal of Project Management, Vol. 21, No. 3, pp.177-187.

Foss, N.J., Husted, K. and Michailova, S. (2010) 'Governing knowledge sharing in organizations: levels of analysis, governance mechanisms, and research directions', Journal of Management Studies, Vol. 47, No. 3, pp.455-482.

Fredriksson, R., Barner-Rasmussen, W. and Piekkari, R. (2006) 'The multinational corporation as a multilingual organization: the notion of a common corporate language', Corporate Communications: An International Journal, Vol. 11, No. 4, pp.406-423.

Gallois, C. and Callan, V.J. (1988) 'Communication accommodation and the prototypical speaker: predicting evaluations of status and solidarity', Language \& Communication, Vol. 8, No. 3, pp.271-283. 


\section{Knowledge sharing and language diversity in organisations}

Gallois, C., Ogay, T. and Giles, H. (2005) 'Communication accommodation theory a look back and a look ahead', in William, G. (Ed.): Theorizing About Intercultural Communication, Sage, pp.121-148.

García, O. and Wei, L. (2013) Translanguaging: Language, Bilingualism and Education, Palgrave Macmillan.

Giles, H. and Coupland, N. (1991) Language: Contexts and Consequences, Thomson Brooks/Cole Publishing Co.

Giles, H. and Powesland, P. (1997) 'Accommodation theory', in Coupland, N. and Jaworski, A. (Eds): Sociolinguistics: A Reader and Coursebook, Macmillan, Basingstoke, pp.232-239.

Gressgård, L.J. (2015) 'Antecedents of knowledge exchange systems usage: motivational and work environment factors', Knowledge and Process Management, Vol. 22, No. 2, pp.112-125.

Hansen, M.T., Mors, M.L. and Lovås, B. (2005) 'Knowledge sharing in organizations: multiple networks, multiple phases', Academy of Management Journal, Vol. 48, No. 5, pp.776-793.

Henderson, J.K. (2005) 'Language diversity in international management teams', International Studies of Management \& Organization, Vol. 35, No. 1, pp.66-82.

Higgins, T. (1999) 'Saying is believing effects: when sharing reality about something biases knowledge and evaluations', in Thompson, L., Levine, J. and Messick, D. (Eds): Shared Cognition in Organisation: The Management of Knowledge, Erlbaum, Mahwah, pp.33-48.

Hinds, P.J., Neeley, T.B. and Cramton, C.D. (2014) 'Language as a lightning rod: power contests, emotion regulation, and subgroup dynamics in global teams', Journal of International Business Studies, Vol. 45, No. 5, pp.536-561.

Holmes, J. and Stubbe, M. (2015) Power and Politeness in the Workplace: A Sociolinguistic Analysis of Talk at Work, Routledge.

Ibarra, H. (1992) 'Homophily and differential returns: sex differences in network structure and access in an advertising firm', Administrative Science Quarterly, Vol. 37, No. 3, pp.422-447.

Ipe, M. (2003) 'Knowledge sharing in organizations: a conceptual framework', Human Resource Development Review, Vol. 2, No. 4, pp.337-359.

Jan, J.M. (2003) 'Code-switching for power wielding: inter-gender discourse at the workplace', Multilingua, Vol. 22, No. 1, pp.41-57.

Jaspers, J. (2010) 'Introduction - society and language use', in Jaspers, J., Verschueren, J. and Östman, J. (Eds): Society and Language Use, John Benjamins Pub. Co, Amsterdam, pp.1-20.

Jørgensen, J.N. (2008) 'Polylingual languaging around and among children and adolescents [Special Issue]', International Journal of Multilingualism, Vol. 5, No. 3, pp.159-275.

Klitmøller, A. and Lauring, J. (2013) 'When global virtual teams share knowledge: media richness, cultural difference and language commonality', Journal of World Business, Vol. 48, No. 3, pp.398-406.

Lauring, J. and Bjerregaard, T. (2007) 'Language use and international business: What can we learn from anthropology?' Hermes-Journal of Language and Communication Studies, Vol. 38, pp.105-118.

Lauring, J. and Selmer, J. (2012) 'International language management and diversity climate in multicultural organizations', International Business Review, Vol. 21, No. 2, pp.156-166.

Lauring, J. and Tange, H. (2010) 'International language management: contained or dilute communication', European Journal of International Management, Vol. 4, No. 4, pp.317-332.

Lønsmann, D. (2011) English as a Corporate Language: Language Choice and Language Ideologies in an International Company in Denmark, Roskilde Universitet.

Makela, K., Kalla, H.K. and Piekkari, R. (2007) 'Interpersonal similarity as a driver of knowledge sharing within multinational corporations', International Business Review, Vol. 16, No. 1, pp.1-22.

Marschan-Piekkari, R., Welch, D. and Welch, L. (1999) 'Adopting a common corporate language: IHRM implications', International Journal of Human Resource Management, Vol. 10, No. 3 , pp.377-390. 


\section{F. Ahmad and G. Widén}

Mcall, C. (2003) 'Language dynamics in the bi- and multilingual workplace', in Bayley, R. and Schecter, S. (Eds): Language Socialization in Bilingual and Multilingual Societies, Multilingual Matters, Clevedon Buffalo, pp.232-250.

McDonald, P. and Tang, Y. (2014) 'Neuroscientific insights into management development theoretical propositions and practical implications', Group \& Organization Management, Vol. 39, No. 5.

Moyer, M. (2008) 'Research as practice: linking theory, method, and data', in Wei, L. and Moyer, M. (Eds): Research Methods in Bilingualism and Multilingualism, John Wiley \& Sons, pp.18-32.

Myers-Scotton, C. (1993) Social Motivations for Codeswitching: Evidence from Africa, Cambridge University Press.

Nonaka, I. and Konno, N. (1998) 'The concept of "ba": building a foundation for knowledge creation', California Management Review, Vol. 40, No. 3, pp.40-54.

Noorderhaven, N. and Harzing, A. (2009) 'Knowledge-sharing and social interaction within MNEs', Journal of International Business Studies, Vol. 40, No. 5, pp.719-741.

Nortier, J. (2008) 'Types and sources of bilingual data', in Wei, L. and Moyer, M. (Eds): Research Methods in Bilingualism and Multilingualism, Wiley-Blackwell, pp.35-52.

Nunan, D. and Carter, R. (2001) Teaching English to Speakers of Other Languages, Cambridge, CUP.

Pardo, J.S., Gibbons, R., Suppes, A. and Krauss, R.M. (2012) 'Phonetic convergence in college roommates', Journal of Phonetics, Vol. 40, No. 1, pp.190-197.

Peltokorpi, V. (2006) 'Knowledge sharing in a cross-cultural context: Nordic expatriates in Japan', Knowledge Management Research \& Practice, Vol. 4, No. 2, pp.138-148.

Radaelli, G., Mura, M., Spiller, N. and Lettieri, E. (2011) 'Intellectual capital and knowledge sharing: the mediating role of organisational knowledge-sharing climate', Knowledge Management Research \& Practice, Vol. 9, No. 4, pp.342-352.

Remennick, L. (2005) 'Resetting the rules of the game: language preferences and social relations of work between Russian immigrants and veteran professionals in an Israeli organization', Journal of International Migration and Integration/Revue de l'integration et de la migration internationale, Vol. 6, No. 1, pp.1-28.

Renzl, B. (2007) 'Language as a vehicle of knowing: the role of language and meaning in constructing knowledge', Knowledge Management Research \& Practice, Vol. 5, No. 1, pp.44-53.

Repko, A.F. (2012) Interdisciplinary Research: Process and Theory, Sage.

Reychav, I. and Te'eni, D. (2009) 'Knowledge exchange in the shrines of knowledge: the "how's" and "where's" of knowledge sharing processes', Computers \& Education, Vol. 53, No. 4, pp.1266-1277.

Ritchie, W.C. and Bhatia, T.K. (2013) 'Social and psychological factors in language mixing', in Bhatia, T. and Ritchie, W. (Eds): The Handbook of Bilingualism and Multilingualism, WileyBlackwell, pp.375-391.

Schomaker, M.S. and Zaheer, S. (2014) 'The role of language in knowledge transfer to geographically dispersed manufacturing operations', Journal of International Management, Vol. 20, No. 1, pp.55-72.

Selmer, J. and Lauring, J. (2015) 'Host country language ability and expatriate adjustment: the moderating effect of language difficulty', International Journal of Human Resource Management, Vol. 26, No. 3, pp.401-420.

Sunaoshi, Y., Kotabe, M. and Murray, J.Y. (2005) 'How technology transfer really occurs on the factory floor: a case of a major Japanese automotive die manufacturer in the United States', Journal of World Business, Vol. 40, No. 1, pp.57-70.

Tan, H. and Gartland, A. (2014) 'Language, knowledge transfer and firm's strategic assets: the strategic role of language in knowledge transfer to China', Journal of Chinese Economic and Business Studies, Vol. 12, No. 1, pp.63-79. 


\section{Knowledge sharing and language diversity in organisations}

Tange, H. and Lauring, J. (2009) 'Language management and social interaction within the multilingual workplace', Journal of Communication Management, Vol. 13, No. 3, pp.218-232.

Thomas, C.A. (2008) 'Bridging the gap between theory and practice: language policy in multilingual organisations', Language Awareness, Vol. 17, No. 4, pp.307-325.

Vaara, E., Tienari, J., Piekkari, R. and Säntti, R. (2005) 'Language and the circuits of power in a merging multinational corporation', Journal of Management Studies, Vol. 42, No. 3, pp.595-623.

Wang, S. and Noe, R.A. (2010) 'Knowledge sharing: a review and directions for future research', Human Resource Management Review, Vol. 20, No. 2, pp.115-131.

Wardhaugh, R. (2010) An Introduction to Sociolinguistics, John Wiley \& Sons.

Wei, L. (2013) 'Codeswithcing', in Bayley, R., Cameron, R. and Lucas, C. (Eds): The Oxford Handbook of Sociolinguistics, Oxford University Press, Oxford, pp.360-378.

Wei, L. and Moyer, M. (2009) Blackwell Guide to Research Methods in Bilingualism and Multilingualism, John Wiley \& Sons.

Welch, D.E. and Welch, L.S. (2008) 'The importance of language in international knowledge transfer', Management International Review, Vol. 48, No. 3, pp.339-360.

West, J. and Graham, J.L. (2004) 'A linguistic-based measure of cultural distance and its relationship to managerial values', Management International Review, Vol. 44, No. 3, pp.239-260.

Yesil, S. (2014) 'Exploring the links among organisational commitment, knowledge sharing and innovation capability in a public organisation', European Journal of International Management, Vol. 8, No. 5, pp.506-527.

Young, V.A. and Martinez, A.Y. (2011) Code-Meshing as World English: Pedagogy, Policy, Performance, National Council of Teachers of English.

Yum, J.O. (1988) 'The impact of Confucianism on interpersonal relationships and communication patterns in east Asia', Communications Monographs, Vol. 55, No. 4, pp.374-388. 